\title{
Violation of majorization relations in entangled states and its detection by means of generalized entropic forms
}

\author{
R. Rossignoli and N. Canosa \\ Departamento de Física, Universidad Nacional de La Plata, C.C. 67, La Plata (1900), Argentina
}

(Received 4 November 2002; published 2 April 2003)

\begin{abstract}
We examine the violation of the majorization relations between the eigenvalues of the full and reduced density operators of entangled states of composite systems and its detection using generalized entropic forms based on arbitrary concave functions. It is shown that the violation of these relations may not always be detected by the conditional von Neumann and Tsallis entropies (for any $q>0$ ). Families of smooth entropic forms which are always able to detect such violations are, however, provided. These features are then examined for particular sets of mixed states in a two-qudit system, which for $d \geqslant 3$ may exhibit different types of violation of the majorization relations. Comparison with the Peres criterion for separability is also shown.
\end{abstract}

DOI: 10.1103/PhysRevA.67.042302

PACS number(s): 03.67.-a, 03.65.Ud, 05.30.- d

Entanglement is now recognized as one of the most important features of quantum mechanics. It represents the possibility of composite quantum systems to exhibit correlations which cannot be accounted for classically. It plays therefore a fundamental role both in philosophical problems concerning the interpretation of quantum theory [1] as well as in applications like quantum computation, quantum cryptography, and quantum teleportation [2-7], where it is actually viewed as a resource. Formally, a quantum system composed of two subsystems $A$ and $B$ is said to be in a separable or unentangled state if the density operator of the whole system can be written as a convex combination of uncorrelated densities [8],

$$
\rho=\sum_{\alpha} \omega_{\alpha} \rho_{A}^{\alpha} \otimes \rho_{B}^{\alpha}, \quad 0 \leqslant \omega_{\alpha} \leqslant 1
$$

where $\Sigma_{\alpha} \omega_{\alpha}=1$ and $\rho_{A}^{\alpha}, \rho_{B}^{\alpha}$ are density operators for each subsystem. Otherwise, the system is said to be entangled or inseparable. When separable, the system fulfills all Bell inequalities and satisfies other properties characteristic of classical composite systems, making it unsuitable for the previous applications. Pure states $(\rho=|\Psi\rangle\langle\Psi|)$ are separable only for tensor product states $\left(|\Psi\rangle=\left|\phi_{A}\right\rangle\left|\phi_{B}\right\rangle\right)$, but in the case of mixed states it is in general much more difficult to determine whether a given density is separable or not. There are several simple necessary conditions for separability [9-14] but they are not sufficient in general.

Among them, one is directly related with disorder and entropy [14]. Let

$$
\rho_{A}=\operatorname{Tr}_{B}[\rho], \quad \rho_{B}=\operatorname{Tr}_{A}[\rho]
$$

be the reduced density matrices of the subsystems, where $\operatorname{Tr}_{A, B}$ denotes partial trace. It has been shown in Ref. [14] that if $\rho$ is separable, then $\rho$ is more mixed (or disordered) than both $\rho_{A}$ and $\rho_{B}$ (the disorder criterion for separability). This property, which can be written as $\rho<\rho_{A}, \rho<\rho_{B}$ means in the present situation that

$$
\mathcal{S}_{i} \equiv \sum_{j=1}^{i} p_{i} \leqslant \mathcal{S}_{i}^{A} \equiv \sum_{j=1}^{i} p_{i}^{A} \quad \text { for } i=1, \ldots, d_{A}
$$

(and similarly $\mathcal{S}_{i} \leqslant \mathcal{S}_{i}^{B}$ for $i=1, \ldots, d_{B}$ ) where $p_{i}, p_{i}^{A}$ are, respectively, the eigenvalues of $\rho$ and $\rho_{A}$ sorted in decreasing order and $d_{A}$ is the dimension of $\rho_{A}$. The set of eigenvalues of $\rho$ is then majorized by those of $\rho_{A}$ and $\rho_{B}$. For a pure state, Eqs. (2) can be fulfilled for $A$ and $B$ only if $p_{1}^{A}$ $=p_{1}^{B}=1$, implying that the state must be a tensor product state. However, for mixed states the disorder criterion is only a necessary condition for separability. No sufficient condition for mixed states can actually be based on the knowledge of the eigenvalues of $\rho$ and $\rho_{A, B}$ alone $[14,15]$. Nevertheless, it is the strongest spectral criterion and expresses a fundamental classical property of separable systems: a classical bipartite system is always more disordered than its subsystems, as the set of joint probabilities $\left\{p_{i j}\right\}$ are always majorized by the set of marginal probabilities $\left\{p_{i}^{A}=\Sigma_{j} p_{i j}\right\}$.

The disorder criterion admits a simple entropic formulation [16]. Let us define the general entropic forms [17]

$$
S_{f}(\rho)=\operatorname{Tr} f(\rho),
$$

where $f$ is an arbitrary smooth concave function satisfying $f(0)=f(1)=0$. These forms satisfy most basic properties of the conventional entropy except those related with additivity [17]. In particular, if $\rho<\rho^{\prime}$, then $S_{f}(\rho) \geqslant S_{f}\left(\rho^{\prime}\right)$ for any $f$ of the previous form $[16,18]$. The disorder criterion implies then that in a separable state, the generalized entropy of the whole system is not less than those of the subsystems:

$$
S_{f}(\rho) \geqslant S_{f}\left(\rho_{A}\right)
$$

and similarly $S_{f}(\rho) \geqslant S_{f}\left(\rho_{B}\right)$. In particular, the standard von Neumann entropy

$$
S(\rho)=-\operatorname{Tr} \rho \ln \rho
$$

corresponds to $f(\rho)=-\rho \ln \rho$, and Eq. (4) implies that the von Neumann conditional entropy $S(\rho)-S\left(\rho_{A}\right)$ [18] is nonnegative in any separable state [11]. Similarly, the Tsallis entropy [19]

$$
S_{q}(\rho)=\left(1-\operatorname{Tr} \rho^{q}\right) /(q-1)
$$


corresponds to $f(\rho)=\left(\rho-\rho^{q}\right) /(q-1)$, which is concave for all $q>0$, and Eq. (2) implies that the Tsallis conditional entropy [20] $\left[S_{q}(\rho)-S_{q}\left(\rho_{A}\right)\right] / \operatorname{Tr} \rho_{A}^{q}$ is non-negative in any separable state. For $q \rightarrow 1, S_{q}(\rho) \rightarrow S(\rho)$.

For a given choice of $f$, Eq. (4) provides therefore a necessary test for separability of mixed states. In the von Neumann case, the criterion is actually rather weak, but in the Tsallis case the validity of Eq. (4) for all $q>0$ provides a more stringent requirement [20-22], which has been shown to yield sufficient conditions for separability for some classes of states, like Werner states for $n$-qubits [20] as well as any two-qubit state diagonal in the Bell basis [16].

Let us come now to the central point of this work. It is also true that if $S_{f}(\rho) \geqslant S_{f}\left(\rho^{\prime}\right)$ for any $f$ of the previous form, then $\rho<\rho^{\prime}$ [18], as will be explicitly shown below. Hence the validity of Eq. (4) for all $f$ (and its partner for $\rho_{B}$ ) provides an equivalent formulation of the disorder criterion. On the other hand, its validity for a particular $f$ does not imply of course that $\rho<\rho_{A}$. Moreover, the validity of Eq. (4) for all $q>0$ in the Tsallis case does not imply $\rho<\rho_{A}$. This may happen whenever the first violation of Eqs. (2) occurs for $i$ $>1$. For sufficiently large $q, S_{q}(\rho) \approx\left(1-m_{1} p_{1}^{q}\right) /(q-1)$, where $m_{1}$ is the multiplicity of the largest eigenvalue $p_{1}$ of $\rho$. Hence, if $p_{1}>p_{1}^{A}, S_{q}(\rho)<S_{q}\left(\rho_{A}\right)$ for sufficiently large $q$. However, if the first violation takes place for $i>1$, Eq. (4) may remain valid for all $q>0$, as can be seen in simple examples. While in a two-qubit system the only possible breakdown of Eqs. (2) is for $i=1$ (as $\mathcal{S}_{2}^{A}=1$ ), this is not the case in systems of larger dimensionality, where it is actually not rare to encounter entangled states in which the first violation of Eqs. (2) occurs for any $i>1$, as shown in the example below. In such cases the Tsallis conditional entropy may not detect entanglement even when the disorder criterion does. The same will hold for any other set of functions $f(p)$ whose maximum is obtained for $p \rightarrow 1$ in the limit where it becomes sharp [17].

We shall now provide a set of smooth concave functions which are always able to detect the breakdown of the inequalities (2), such that $S_{f}(\rho)<S_{f}\left(\rho_{A}\right)$ for some $f$ of this set if Eqs. (2) are violated for some $i \geqslant 1$. This can be achieved with functions having a maximum at an optimizable point $\alpha \in(0,1)$, which can be made arbitrarily sharp by adjusting a second parameter. A possibility is

$$
f(\rho)=g_{t}(\rho-\alpha)-(1-\rho) g_{t}(-\alpha)-\rho g_{t}(1-\alpha),
$$

where $\alpha \in[0,1]$ and $g_{t}(x)$ is a smooth concave function satisfying

$$
\lim _{t \rightarrow \infty} g_{t}(x)=-|x| / 2
$$

An example is (see Fig. 1)

$$
g_{t}(x)=-(2 t)^{-1} \ln \cosh (t x) .
$$

For any of these functions, $f(0)=f(1)=0$, while for $t \rightarrow \infty$ and $0<\alpha<1$,

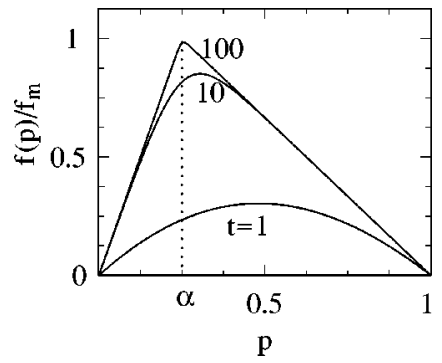

FIG. 1. The entropic functions (7) with $g$ given by Eq. (8), for $\alpha=1 / 4$ and different values of $t . f_{m}=\alpha(1-\alpha)$ denotes the maximum value in the $t \rightarrow \infty$ limit.

$$
f(p) \rightarrow \begin{cases}p(1-\alpha), & p \leqslant \alpha \\ \alpha(1-p), & p \geqslant \alpha .\end{cases}
$$

In this limit,

$$
\begin{aligned}
S_{f}(\rho) & =\operatorname{Tr}\left[g_{t}(\rho-\alpha)\right]-(D-1) g_{t}(-\alpha)-g_{t}(1-\alpha) \\
& \rightarrow \alpha\left(n_{\alpha}-1\right)+1-\sum_{p_{i}>\alpha} p_{i},
\end{aligned}
$$

where $D=d_{A} d_{B}$ is the dimension of $\rho$ and $n_{\alpha}$ is the number of eigenvalues of $\rho$ larger than $\alpha$. It is now seen that if $\rho$ $K \rho_{A}, S_{f}(\rho)<S_{f}\left(\rho_{A}\right)$ for some $\alpha$ and $t$. Suppose that the inequalities (2) are first violated for $i=j$, i.e., $\mathcal{S}_{j}>\mathcal{S}_{j}^{A}$, which implies $p_{j}>p_{j}^{A}$. Choosing $\alpha=p_{j}^{A}$, we have $n_{\alpha}(\rho)$ $=j^{\prime} \geqslant j$, obtaining, for $t \rightarrow \infty$,

$$
\begin{aligned}
S_{f}(\rho)-S_{f}\left(\rho_{A}\right) & \rightarrow p_{j}^{A}\left(j^{\prime}-j\right)-\left(\mathcal{S}_{j^{\prime}}-\mathcal{S}_{j}^{A}\right) \\
& \leqslant-\left[\left(p_{j^{\prime}}-p_{j}^{A}\right)\left(j^{\prime}-j\right)+\mathcal{S}_{j}-\mathcal{S}_{j}^{A}\right]<0 .
\end{aligned}
$$

Hence $S_{f}(\rho)<S_{f}\left(\rho_{A}\right)$ for sufficiently large $t$ and $\alpha$ sufficiently close to $p_{j}^{A}$.

In the opposite limit $t \rightarrow 0^{+}$, and assuming $g_{t}(x)$ $=h(t x) / t$, as in Eq. (8), $f(\rho) \rightarrow t\left|h^{\prime \prime}(0)\right|\left(\rho-\rho^{2}\right) / 2$, becoming hence independent of $\alpha$ and proportional to the Tsallis case $q=2$. On the other hand, for $\alpha=0$ or 1 and $t \rightarrow \infty$, the lowest nonzero order of $f$ depends on the higher-order term $r_{t}(x)=g_{t}(x)+|x| / 2-g_{t}(0)$. For instance, if $\alpha=1, f(\rho)$ $=r_{t}(\rho-1)-(1-\rho) r_{t}(-1)$, with $\quad r_{t}(x)=-(2 t)^{-1} \ln [(1$ $\left.\left.+e^{-2 t|x|}\right) / 2\right]$ in the case (8).

Example. Let us consider a system of two qudits ( $d$-dimensional subsystems) with $d \geqslant 3$. We will first examine a mixture of two antisymmetric states with a full random state,

$$
\rho=x_{1}\left|01^{-}\right\rangle\left\langle 01^{-}\left|+x_{2}\right| 02^{-}\right\rangle\left\langle 02^{-}\right|+y I_{A} \otimes I_{B},
$$

where $\left|i j^{-}\right\rangle=(|i j\rangle-|j i\rangle) / \sqrt{2}, y=\left(1-x_{1}-x_{2}\right) / d^{2}$ and $I_{A, B}$ $=\sum_{i=0}^{d-1}|i\rangle\langle i|$. For $x_{2}=0$ and $d=2$, it reduces to a WernerPopescu state for two qubits [8,23]. Its eigenvalues are obviously $x_{1}+y, x_{2}+y$ and $y\left[\left(d^{2}-2\right)\right.$-fold degenerate $]$. Positivity of $\rho$ imposes then the conditions $x_{1}+x_{2} \leqslant 1, x_{i} \leqslant\left(d^{2}\right.$ $-1) x_{j}+1, i \neq j$, which determine a triangle $R$ with vertices at $\boldsymbol{x} \equiv\left(x_{1}, x_{2}\right)=(1,0),(0,1),-(1,1) /\left(d^{2}-2\right)$, shown in Fig. 2 for $d=3$. Negative values of $x_{i}$ are in principle feasible 


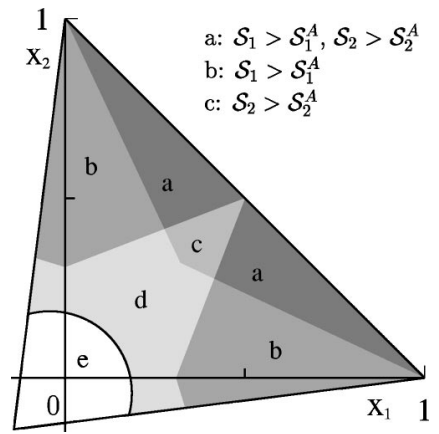

FIG. 2. Region of allowed values of $x_{1}, x_{2}$ in Eq. (11), for $d$ $=3 . \rho$ is entangled in sectors $a, b, c, d$, and separable in $e$. The disorder criterion detects entanglement just in sectors $a, b, c$, where the inequalities (2) that are not fulfilled are indicated.

and represent a depletion of the antisymmetric states with respect to the fully mixed state.

The lowest eigenvalue of the partial transpose of $\rho$ is

$$
\sigma(x)=y-|x| / 2,
$$

where $|\boldsymbol{x}|=\left(x_{1}^{2}+x_{2}^{2}\right)^{1 / 2}$. The equation $\sigma(\boldsymbol{x})=0$ determines an ellipse centered at $x_{1}=x_{2}=-4 /\left(d^{4}-8\right)$, with axes rotated 45 degrees with respect to the $x_{1}, x_{2}$ axes, and $\sigma(\boldsymbol{x})$ $<0$ outside this ellipse. Hence, according to the Peres criterion [9], $\rho$ will be entangled in the intersection of this region with $R$ (see Fig. 2). Moreover, it can be easily shown that $\rho$ is indeed separable if $\sigma(\boldsymbol{x}) \geqslant 0$. We may rewrite Eq. (11) in the form

$$
\begin{aligned}
\rho & =\sum_{i=1}^{n} \rho_{i}+y\left(I_{A} \otimes I_{B}-\sum_{i=1}^{n} Q_{i}\right), \\
\rho_{i} & \equiv x_{i}\left|0 i^{-}\right\rangle\left\langle 0 i^{-}\right|+y Q_{i}, \\
Q_{i} & =q_{i}|00\rangle\langle 00|+| 0 i\rangle\langle 0 i|+| i 0\rangle\langle i 0|+| i i\rangle\langle i i|,
\end{aligned}
$$

where $n=2, q_{i} \geqslant 0, q_{1}+q_{2}=1$. For $x_{1}, x_{2} \in R, \rho_{i}$ is a nonnegative operator for a two-qubit system, where the Peres criterion is sufficient [10], and will then be separable if and only if the lowest eigenvalue of its partial transpose is nonnegative. This leads to the condition

$$
x_{i}^{2} \leqslant 4 q_{i} y^{2} .
$$

If Eq. (16) holds for $i=1,2$, then $|\boldsymbol{x}|^{2} \leqslant 4 y^{2}$, implying $\sigma(\boldsymbol{x})$ $\geqslant 0$. Moreover, choosing $q_{i}=x_{i}^{2} /|\boldsymbol{x}|^{2}$, it is seen that any state satisfying $\sigma(\boldsymbol{x}) \geqslant 0$ will fulfill Eqs. (16) for $i=1,2$, implying separability of $\rho_{i}$ and hence of $\rho$. Thus, $\rho$ is entangled if and only if $\sigma(\boldsymbol{x})<0$. Denoting with $\gamma$ the angle between $\boldsymbol{x}$ and $\boldsymbol{v}=(1,1)$, this implies entanglement for

$$
|\boldsymbol{x}|>\left[\sqrt{2} \cos \gamma+d^{2} / 2\right]^{-1} .
$$

The entanglement threshold for $|\boldsymbol{x}|$ is minimum at $\gamma=0$ $\left(x_{1}=x_{2}\right.$ ), and decreases as $d^{-2}$ as $d$ increases (for $d=3$ the entangled region already covers $87 \%$ of $R$ ). If $x_{2}=0 \mathrm{Eq}$. (17) implies entanglement just for

$$
x_{1}>\left(1+d^{2} / 2\right)^{-1} \text {. }
$$

For $d=2$ this yields $\left|x_{1}\right|>1 / 3$, in agreement with the wellknown result for a Werner-Popescu state $[7,9]$.

As seen in Fig. 2, the disorder criterion detects entanglement only in a sector of the entangled region (covering $77 \%$ of it for $d=3$ ), which can be classified according to the subset of inequalities (2) that are violated. Note that the complement of this sector is not convex. The reduced density matrix of any of the subsystems is

$$
\rho_{A}=\frac{x_{1}+x_{2}}{2}|0\rangle\left\langle 0\left|+\frac{x_{1}}{2}\right| 1\right\rangle\left\langle 1\left|+\frac{x_{2}}{2}\right| 2\right\rangle\langle 2|+y d I_{A} .
$$

Hence, for $x_{1} \geqslant x_{2} \geqslant 0, \mathcal{S}_{1}>\mathcal{S}_{1}^{A}$ if $x_{1}+y>\left(x_{1}+x_{2}\right) / 2+y d$, i.e.,

$$
x_{1}>\frac{1+x_{2}(\delta-1)}{\delta+1}, \quad \delta \equiv \frac{d^{2}}{2(d-1)},
$$

while if $x_{1} \geqslant 0 \geqslant x_{2}$, this occurs for $x_{1}+y>x_{1} / 2+y d$, i.e., $x_{1}>\left(1-x_{2}\right) /(1+\delta)$. Besides, for $x_{1} \geqslant x_{2}, \mathcal{S}_{2}>\mathcal{S}_{2}^{A}$ in $R$ just for $x_{1}+x_{2}+2 y>x_{1}+x_{2} / 2+2 y d$, i.e.,

$$
x_{1}>1-x_{2}(1+\delta / 2) \text {. }
$$

These regions partially overlap, giving rise, together with their symmetric partners for $x_{2}>x_{1}$, to the sectors $a, b, c$ of Fig. 2. In $c$, only the second inequality is violated. For 3 $\leqslant i \leqslant d, \mathcal{S}_{i} \leqslant \mathcal{S}_{i}^{A} \forall x_{1}, x_{2} \in R$, with $\mathcal{S}_{i}=\mathcal{S}_{i}^{A}$ only along the border $x_{1}+x_{2}=1$ (where $\mathcal{S}_{i}=1$ for $i \geqslant 2$ ).

In particular, for $x_{2}=0$ the disorder criterion predicts entanglement just for

$$
x_{1}>(1+\delta)^{-1} \text {, }
$$

which provides a bound that is strictly larger than Eq. (18) except for $d=2$, and decreases only as $d^{-1}$ as $d$ increases. The same occurs for $x_{1}=x_{2}(\gamma=0)$, where Eq. (20) predicts entanglement just for $|\boldsymbol{x}|>[\sqrt{2}+\delta /(2 \sqrt{2})]^{-1}$.

For any entropic function $f$, the region where Eq. (4) does not hold, i.e., where

$$
S_{f}^{A}(\rho) \equiv S_{f}(\rho)-S_{f}\left(\rho_{A}\right)
$$

is negative, will be necessarily contained in sectors $a, b, c$. As seen in Fig. 3 for $d=3$, this region is rather small for the von Neumann entropy $(q=1)$, but in the Tsallis case it increases as $q$ increases, covering sectors $a$ and $b$ for $q \rightarrow \infty$, although it never covers sector $c$. Even the region where $S_{f}^{A}(\rho)<0$ for some $q>0$, depicted in the bottom panel, leaves out an appreciable fraction of sector $c$. The border of this region in sector $c$ corresponds to finite and varying values of $q$, which can be determined by the simultaneous conditions $S_{f}^{A}(\rho)=0, \partial S_{f}^{A}(\rho) / \partial q=0$. Note also that along the outer border $\left(x_{1}+x_{2}=1\right), S_{f}^{A}(\rho) \leqslant 0$ for any $f$ since here $\rho^{A}<\rho$. As $q \rightarrow 0^{+}$, the region where $S_{f}^{A}(\rho)<0$ shrinks and approaches this line.

The typical behavior of the Tsallis conditional entropy $\overline{S_{f}^{A}}(\rho) \equiv S_{f}^{A}(\rho) / \operatorname{Tr}\left[\rho_{A}^{q}\right]$ in sector $c$ is depicted in the top panel 

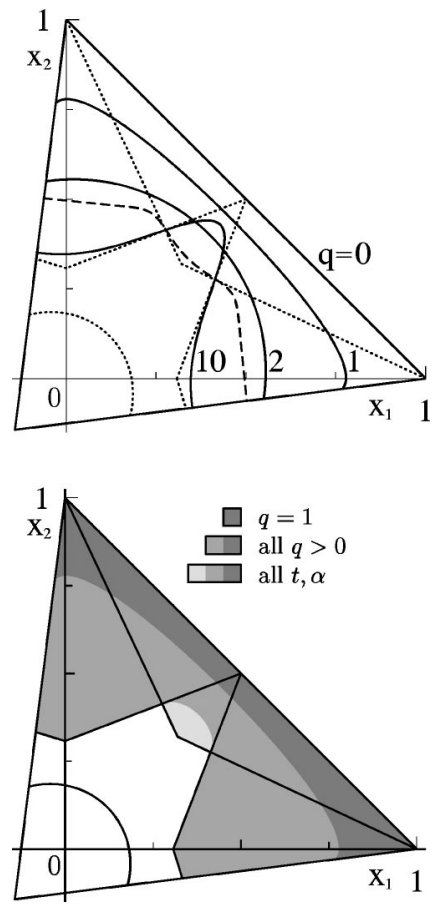

FIG. 3. Top: curves where $S_{f}^{A}(\rho)=0$ according to the Tsallis entropy for the indicated values of $q$ (solid lines), and the function (7) (dashed line) with $g$ given by Eq. (8) and $\alpha=0.28, t=30$. The curve for $q=1$ corresponds to the von Neumann entropy. Dotted lines indicate the boundaries of the sectors of Fig. 2. Bottom: union of sectors where $S_{f}^{A}(\rho)<0$ according to the von Neumann entropy $(q=1)$, the whole set of Tsallis entropies (all $q>0)$, and the whole set of functions (7) and (8) $(t>0,0<\alpha<1)$.

of Fig. 4 as a function of $q$. Here $S_{f}^{A}(\rho)$ may become negative only in a finite interval of $q$ values. For $x_{1}=x_{2}=x$, this occurs just for $x>0.381$ [at this point $S_{f}^{A}(\rho)=0$ just for $q$ $=3.575]$. In comparison, along this line the disorder criterion predicts entanglement for $x>(2+\delta / 2)^{-1}=0.32$, while the true value given by the Peres criterion is $x>(2$ $\left.+d^{2} / \sqrt{2}\right)^{-1} \approx 0.12$ [in the von Neumann case, $S_{f}^{A}(\rho)<0$ just for $x>0.452]$. Note as well that $\overline{S_{f}^{A}}(\rho)$ does not necessarily develop a local minimum as a function of $q$ in this sector (for $x_{1}=x_{2}=x$ this occurs just for $\left.x>0.334\right)$.

In the case of the functions (7), $S_{f}^{A}(\rho)$ becomes negative for sufficiently large $t$ at any point of sectors $a, b, c$ if $\alpha$ is appropriately chosen. Due to the symmetries of the present case, just two values of $\alpha$ are required: For $d=3$, in sectors $a, b$ it is sufficient to choose any single $\alpha>5 / 13 \approx 0.385$, which is the value of $p_{1}^{A}$ at the tip of sector $b[\boldsymbol{x}$ $=(4 / 13,0)]$, whereas the whole sector $c$ can be covered choosing $\alpha=7 / 25=0.28$, which is the value of $p_{2}^{A}$ at the inner tip of this sector $\left(x_{1}=x_{2}=0.32\right)$. In the case (8), the curves where $S_{f}^{A}(\rho)=0$ are, for large $t$ and $\alpha>1 / 2$, similar to those of the Tsallis case for large $q$, whereas if $\alpha=0.28$, they approach for large $t$ the inner border of sector $c$, as seen in the top panel of Fig. 3. The behavior of $\overline{S_{f}^{A}}(\rho)$ $\equiv S_{f}^{A}(\rho) /\left|\operatorname{Tr}\left[g_{t}\left(\rho_{A}\right)\right]\right|$ in sector $c$ is depicted in Fig. 4, where it is seen that for sufficiently large $t$, it becomes negative in a finite interval of $\alpha$ values (which always includes $\alpha$ $=0.28$ ), for any $x>0.32$.
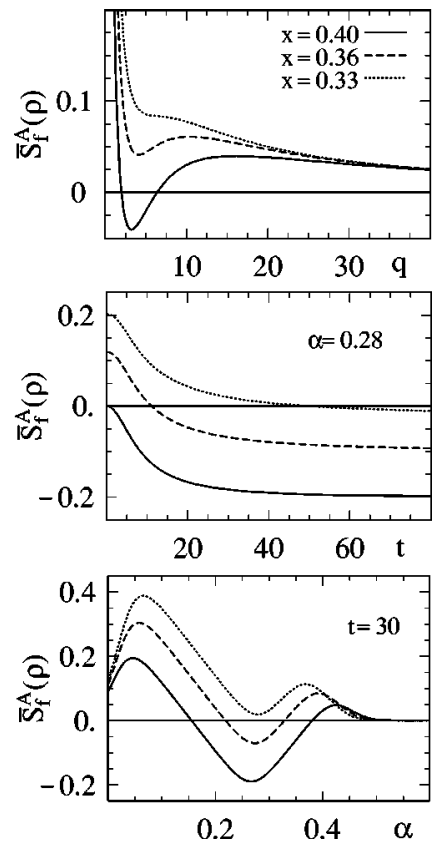

FIG. 4. The behavior of $\overline{S_{f}^{A}}(\rho)$ for $x_{1}=x_{2}=x$ (sector $c$ of Fig. 2) at the indicated values of $x$, according to the Tsallis entropy as a function of $q$ (top), and the function (7) for the case (8), as a function of $t$ (center) and $\alpha$ (bottom).

Similar considerations hold for the states

$$
\rho=\sum_{i=1}^{n} x_{i}\left|0 i^{-}\right\rangle\left\langle 0 i^{-}\right|+y I_{A} \otimes I_{B}
$$

where $n \leqslant d-1$ and $y=\left(1-\sum_{i=1}^{n} x_{i}\right) / d^{2}$. Its eigenvalues are $x_{i}+y, i=1, \ldots, n$, and $y\left[\left(d^{2}-n\right)\right.$-fold degenerate $]$, and the allowed values of $x_{i}$ are contained in a region $R$ bounded by the conditions $y \geqslant 0, x_{i} \geqslant-y$.

The lowest eigenvalue of the partial transpose is again given by Eq. (12), with $|\boldsymbol{x}|=\left[\sum_{i=1}^{n} x_{i}^{2}\right]^{1 / 2}$, and it can be seen that $\rho$ is entangled if and only if $\sigma(x)<0$ : writing $\rho$ in the form (13), with $q_{i} \geqslant 0, \sum_{i=1}^{n} q_{i}=1$, any state satisfying $\sigma(\boldsymbol{x}) \geqslant 0$ is seen to fulfill Eqs. (16) for $i=1, \ldots, n$ if $q_{i}$ $=x_{i}^{2} /|\boldsymbol{x}|^{2}$, which implies separability of $\rho_{i}$ and hence, of $\rho$. The entanglement condition $\sigma(\boldsymbol{x})<0$ can be cast as

$$
|\boldsymbol{x}|>\left(\sqrt{n} \cos \gamma+d^{2} / 2\right)^{-1},
$$

where $\gamma$ is the angle between $\boldsymbol{x}$ and $\boldsymbol{v}=(1, \ldots, 1)$, and corresponds to the region outside an $n$-dimensional ellipsoid. For $|\gamma|<\pi / 2$ and fixed $d$, the entanglement threshold for $|\boldsymbol{x}|$ decreases as $n$ increases.

For $n \geqslant 5$ (and $d \geqslant n+1$ ) a novel feature arises: entanglement may also occur for $x_{i}<0 \forall i$, i.e., in the vicinity of $\gamma$ $=\pi$, since the vertex of $R$ in this direction $\left(x_{v i}=-\left(d^{2}\right.\right.$ $-n)^{-1}$ for $\left.i=1, \ldots, n\right)$ lies outside the ellipsoid, $\left|\boldsymbol{x}_{v}\right|$ $=\sqrt{n} /\left(d^{2}-n\right)>\left(d^{2} / 2-\sqrt{n}\right)^{-1}$ for $n>4$. Thus, for $n \geqslant 5$ entanglement can also occur by depletion of antisymmetric states, although the corresponding region is very small (see Fig. 5 for the case $n=5, d=6$ ). Nevertheless, the ellipsoid 


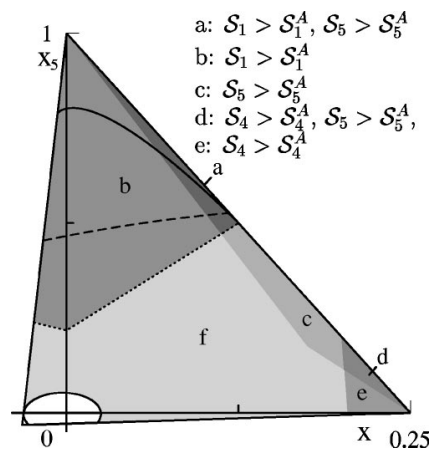

FIG. 5. Section of allowed values of $x, x_{5}$ in the state (23) for $d=6, n=5$, and $x_{i}=x$ for $i=1, \ldots, 4 . \rho$ is entangled in all shaded sectors. The disorder criterion detects entanglement in sectors $a, b, c, d, e$, where the inequalities (2) that are not fulfilled are indicated. Also depicted are the curves where $S_{f}^{A}(\rho)=0$ in the von Neumann case (solid line) and the Tsallis case $q=2$ (dashed line), such that $S_{f}^{A}(\rho)<0$ above these curves. The dotted line indicates the boundary of the region covered by all $q>0$ in the Tsallis case (see text).

$\sigma(x) \geqslant 0$ is never fully contained in $R$. Points of the boundary of $R$ like $x_{j}=0$ if $j \neq i, x_{i}=-1 /\left(d^{2}-1\right)$, fulfill $\sigma(\boldsymbol{x})$ $>0 \quad \forall d \geqslant 2$.

The disorder criterion ensures again entanglement in a much smaller region. Moreover, the first violation of the inequalities (2) may now occur for any $i$ between 1 and $n$. The reduced density of system $A$ is

$$
\rho_{A}=\frac{1}{2} \sum_{i=1}^{n} x_{i}[|i\rangle\langle i|+| 0\rangle\langle 0|]+y d I_{A} .
$$

For $x_{1} \geqslant x_{2} \geqslant \cdots \geqslant x_{n} \geqslant 0$ and $1 \leqslant i \leqslant n, \mathcal{S}_{i}>\mathcal{S}_{i}^{A}$ for

$$
x_{i}>\frac{1}{2} \sum_{j=i}^{n} x_{j}+i y(d-1) .
$$

According to the values of the $x_{i}$, we may have violation of the inequalities (2) for any set of $m$ distinct indices $i_{j}$, with $m \leqslant n, i_{j} \leqslant n$. In particular, if $x_{i}=x \geqslant 0$ for $i=1, \ldots, n(\gamma$ $=0$ ), only the $n$th inequality can be violated: $\mathcal{S}_{n}>\mathcal{S}_{n}^{A}$ for

$$
x>n /\left(n^{2}+\delta\right),
$$

i.e., $|\boldsymbol{x}|>\left[\sqrt{n}+\delta / n^{3 / 2}\right]^{-1}$. This bound is larger than that given by Eq. (24) for $\gamma=0$, for any $n \geqslant 1, d \geqslant 3$. On the other hand, the disorder criterion is not able to predict entanglement for $x<0(\gamma=\pi)$ for any $d, n$.
For instance, Fig. 5 depicts, for $n=5$ and $d=6$, the intersection of $R$ with the plane $x_{i}=x$ for $i=1, \ldots, 4$. The separable sector covers only a small fraction of this section $(\sim 2,6 \%)$ but the disorder criterion covers only $\approx 51 \%$ of the entangled region. Moreover, the first violation of the inequalities (2) may occur for $i=1,4$ or 5 , the last two in sectors $c, d, e$, although the largest region corresponds to $i$ $=1$ (sectors $a, b$, accounting for $40 \%$ of the entangled region).

The region where $S_{f}^{A}(\rho)<0$ is small in the von Neumann case, but increases as $q$ increases in the Tsallis case, covering sectors $a$ and $b$ for $q \rightarrow \infty$, although the covering of sectors $c, d, e$ is very poor. The inner border of the region where $S_{f}^{A}(\rho)<0$ for some $q>0$ lies very close to the outer boundary $\left(\sum_{i=1}^{n} x_{i}=1\right)$ in these sectors, being indistinguishable from it in the scale of the figure. For instance, for $x_{5}=x$ (sector $c$ ), the boundary lies at $x=0.2$ and $S_{f}^{A}(\rho)<0$ for some $q$ just for $x>0.19997$, while for $x_{5}=0$ (sector $e$ ), the boundary is at $x=0.25$ and $S_{f}^{A}(\rho)<0$ only for $x>0.2492$. In comparison, along these lines the disorder criterion predicts entanglement for $x>0.175$ and $x>0.204$, respectively, as given by Eq. (26). For the function (7), it is necessary to employ in this case an interval of $\alpha$ values in order to have $S_{f}^{A}(\rho)<0$ for large $t$ in all points of regions $c, d, e(\alpha$ $\in[0.069,0.108]$ in $c$ and $\alpha \in[0.114,0.134]$ in $e$ ), although for the whole sectors $a$ and $b$ it is sufficient to take a single $\alpha>1 / 4$. It should be mentioned that all previous equations and results remain valid if the antisymmetric states in Eqs. (11) or (23) are replaced by symmetric states $\left|i j^{+}\right\rangle=(|i j\rangle$ $+|j i\rangle) / \sqrt{2}$.

In conclusion, we have provided an example of a set of smooth entropic forms which are always able to detect the breakdown of the majorization relations (2) in entangled states, through the sign of the entropic difference (22). We have also shown that when the first violation occurs for $i$ $>1$, the Tsallis conditional entropy may not detect such violations for any $q$, being hence weaker in these cases than the disorder criterion. The present entropic forms are therefore necessary for detecting and measuring entanglement by entropic means in such situations. These issues have been illustrated in detail for particular states of a two-qudit system, which show that the first violation of Eqs. (2) may in fact occur for any $i \leqslant d-1$.

R.R. and N.C. acknowledge support from CIC and CONICET, respectively, of Argentina, and a grant of Fundación Antorchas.
[1] E. Schrödinger, Naturwissenschaften 23, 807 (1935); Proc. Cambridge Philos. Soc. 31, 555 (1935).

[2] D.P. DiVincenzo, Science 270, 255 (1995); C.H. Bennett, D.P. DiVincenzo, J.A. Smolin, and W.K. Wootters, Phys. Rev. A 54, 3824 (1996).

[3] A.K. Ekert, Phys. Rev. Lett. 67, 661 (1991); Nature (London) 358, 14 (1992).
[4] M. Nielsen and I. Chuang, Quantum Computation and Quantum Information (Cambridge University Press, Cambridge, 2000).

[5] A. Galindo and M.A. Martín Delgado, Rev. Mod. Phys. 74, 347 (2002).

[6] C.H. Bennett et al., Phys. Rev. Lett. 70, 1895 (1993).

[7] C.H. Bennett et al., Phys. Rev. Lett. 76, 722 (1996). 
[8] R.F. Werner, Phys. Rev. A 40, 4277 (1989).

[9] A. Peres, Phys. Rev. Lett. 77, 1413 (1996).

[10] M. Horodecki, P. Horodecki, and R. Horodecki, Phys. Lett. A 223, 1 (1996).

[11] R. Horodecki and M. Horodecki, Phys. Rev. A 54, 1838 (1996).

[12] M. Horodecki and P. Horodecki, Phys. Rev. A 59, 4206 (1999).

[13] N.J. Cerf, C. Adami, and R.M. Gingrich, Phys. Rev. A 60, 898 (1999).

[14] M.A. Nielsen and J. Kempe, Phys. Rev. Lett. 86, 5184 (2001).

[15] K.G.H. Vollbrecht and M.M. Wolf, e-print quant-ph/0107014.

[16] R. Rossignoli and N. Canosa, Phys. Rev. A 66, 042306 (2002).
[17] N. Canosa and R. Rossignoli, Phys. Rev. Lett. 88, 170401 (2002); R. Rossignoli and N. Canosa, Phys. Lett. A 264, 148 (1999).

[18] A. Wehrl, Rev. Mod. Phys. 50, 221 (1978).

[19] C. Tsallis, J. Stat. Phys. 52, 479 (1988).

[20] S. Abe and A.K. Rajagopal, Physica A 289, 157 (2001); S. Abe, Phys. Rev. A 65, 052323 (2002).

[21] C. Tsallis, S. Lloyd, and M. Baranger, Phys. Rev. A 63, 042104 (2001).

[22] C. Tsallis, D. Prato, and C. Anteneodo, Eur. Phys. J. B 29, 605 (2002).

[23] S. Popescu, Phys. Rev. Lett. 72, 797 (1994). 